\title{
Administración electrónica y gestión documental. Consideraciones a la luz de la Ley para el Acceso Electrónico de los Ciudadanos a los Servicios Públicos
}

\author{
Por Carlota Bustelo $y$ Elisa García-Morales
}

Resumen: La entrada en vigor de la Ley para el Acceso Electrónico de los Ciudadanos a los Servicios Públicos va a tener un gran impacto sobre las administraciones públicas en España. Esta ley afecta muy directamente a la gestión de documentos en el ámbito de las administraciones, eliminando las posibles incertidumbres sobra la validez de los documentos electrónicos. Se argumenta porque la implantación de un sistema de gestión de documentos electrónicos es necesaria para poder cumplir con esta ley. Como herramienta práctica, se enumeran en forma de preguntas las cuestiones a resolver sobre gestión documental cuando se produce una implantación por procesos o trámites de la e-administración.

Palabras clave: E-administración, Legislación, Gestión de documentos electrónicos, España

Title: Electronic administration, records management, and Spain's Electronic Access to Public Services legislation

Abstract: Implementation of the e-government law guaranteeing Spanish citizens electronic access to public services (Ley para el Acceso Electrónico de los Ciudadanos a los Servicios Públicos) will have great impact on public administration at every level. This law directly affects government records and document management, removing possible uncertainties about the validity of electronic records. Arguments are offered concerning the need for a document and records management system to support com-
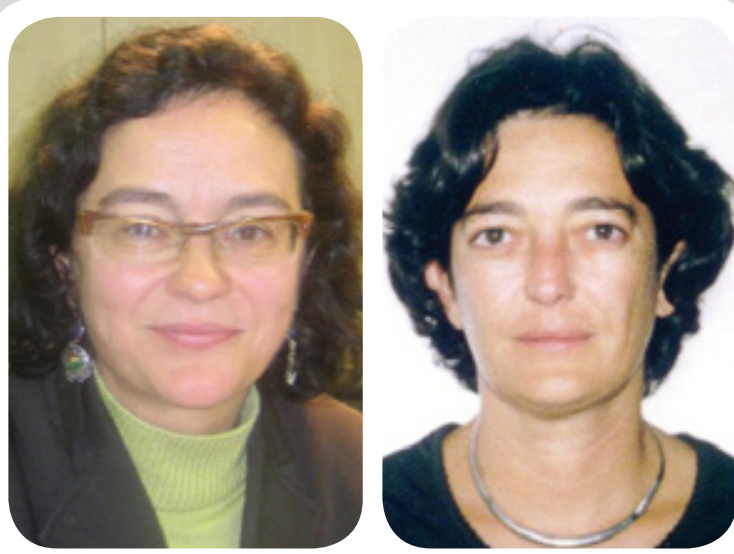

Carlota Bustelo Ruesta y Elisa García-Morales Huidobro son socias-directoras de Inforárea SL, empresa consultora especializada en la organización de la documentación y la información. Han dirigido numerosos proyectos de gestión documental, organización de contenidos y diseño de sistemas de información en España y Latinoamérica, temas sobre los que han publicado artículos en congresos y revistas profesionales. Una de sus especialidades es la gestión de documentos electrónicos en las organizaciones, terreno en el que actualmente dirigen varios proyectos de evaluación, diseño e implantación. Inforárea es miembro activo del CTN50 (Información y Documentación) de Aenor y de su Subcomité Gestión de Documentos y Aplicaciones. Recientemente Carlota ha sido elegida "convenor" de grupo de trabajo internacional de ISO que se encargará de la redacción de la nueva norma de requerimientos para la gestión de documentos. pliance with the law. As a practical tool, a list of questions is provided to help resolve records and document management issues when e-government procedures or business processes are implemented.

Keywords: E-government, Legislation, Electronic records management, Spain

Bustelo, Carlota; García-Morales, Elisa. “Administración electrónica y gestión documental. Consideraciones a la luz de la Ley para el Acceso Electrónico de los Ciudadanos a los Servicios Públicos”. En: El profesional de la información, 2008, enero-febrero, v. 17, n. 1, pp. 106-111.

DOI: 10.3145/epi.2008.ene.13

1. Una nueva ley de gran impacto

EL DÍA 23 DE JUNIO DE 2007 se publicó en el Boletín Oficial del Estado (BOE) la Ley para el acceso electrónico de los ciudadanos a los servicios públicos (conocida como la ley de la e-administración) que reconoce el derecho de los ciudadanos a exigir a las administraciones públicas -en cualquiera de sus niveles- la realización de los trámites por medios electrónicos.
Las administraciones deberán poner en marcha las medidas necesarias para que ello sea una realidad a partir del 31 de diciembre de 2009. Con este nuevo texto legal se pretende fomentar la realización de tramitaciones administrativas por internet, teléfono, televisión o cualquier otro medio electrónico disponible en el futuro.

En este texto queremos llamar la atención sobre el impacto que esta disposición tiene en la gestión documental de las administraciones públicas, pues respalda jurídicamente la validez de los documentos electrónicos.

La administración electrónica es una realidad imparable y muchas instituciones y organismos públicos están inmersos desde hace años en proyectos para hacerla posible. En España, gran parte de estas iniciativas se han focalizado en el "frontoffice" y han tenido poco impacto en la manera de trabajar con los do- 
cumentos por parte de las administraciones. La inseguridad respecto a la validez de los documentos electrónicos y -hasta fecha reciente- la escasa utilización de la firma electrónica, son los motivos aducidos para que los documentos que dan soporte a los trámites y procedimientos se continúen gestionando en papel y en paralelo a los procesos automatizados y documentos electrónicos cuando éstos existen. La mayoría de los expedientes y documentos se siguen conservando de forma tradicional.

Algunas iniciativas de digitalización llevadas a cabo han servido para crear copias de originales existentes en papel, con el propósito de facilitar el acceso y uso; pero en ningún caso han suplantado a éstos pues nadie se ha atrevido a eliminar el papel.

Sí hay casos en que las aplicaciones informáticas han hecho "desmaterializarse" determinados documentos que tradicionalmente se venían produciendo y conservando en soporte en papel: véase por ejemplo el padrón municipal o los libros de cuentas. Esto presenta otro tipo de problemática -a la que se ha prestado escasa atención- que es la conservación de esta información, que antes se plasmaba en documentos y ahora constituyen evidencias electrónicas contenidas en bases de datos.

Con la introducción del registro y las notificaciones telemáticas (Ley 24/2001) y el uso la firma electrónica (Ley 59/2003) muchas organizaciones ya se han encontrado con documentos electrónicos originales $\mathrm{y}$ auténticos, pero que forman a su vez parte de trámites y expedientes en los que el resto de la documentación está en papel, lo cual prácticamente obliga a su impresión para poderlos gestionar.

\section{Qué cambia con la Laecsp ${ }^{1}$} para la gestión documental

La Ley consagra el derecho de los ciudadanos a relacionarse con las administraciones por medios electrónicos y obliga a las administraciones a poner los medios para hacer posible entre otras cosas:

"presentar solicitudes y recursos; realizar el trámite de audiencia cuando proceda; efectuar pagos o acceder a las notificaciones y comunicaciones que les remitan la Administración Pública” (Exposición de motivos I)

Esto lleva implícito un crecimiento exponencial de masa de documentación que existirá ya en su forma original en soporte electrónico. La gestión documental electrónica se hace imprescindible para hacer frente a este problema.

La Laecsp acaba con la indefinición en cuanto a la validez de los documentos electrónicos, consagrando la existencia tanto del documento como del expediente electrónico (Capítulo IV, art. 29, 32), y el acceso de los ciudadanos al mismo:

“...los interesados en un procedimiento tienen derecho de acceso al mismo y ver los documentos. Lo mismo debe suceder, como mínimo, en un expediente iniciado electrónicamente o tramitado de esta forma. Dicho expediente debe poder permitir el acceso en línea a los interesados para verificar la situación del expediente, sin mengua de todas las garantías de la privacidad." (Exposición de motivos III)

La Ley establece las modalidades de copias de los documentos electrónicos: copias digitalizadas de documentos que originalmente existían en soporte papel y viceversa, copias en papel de documentos cuya existencia original es en soporte electrónico (Capítulo IV, art. 30. Copias electrónicas).

La Ley respalda el archivo electrónico de documentos siempre que esté sujeto a unas medidas de segu- ridad que garanticen la integridad, autenticidad, confidencialidad, calidad, protección y conservación de los documentos almacenados.

"Artículo 31. Archivo electrónico de documentos.

Podrán almacenarse por medios electrónicos todos los documentos utilizados en las actuaciones administrativas.

Los documentos electrónicos que contengan actos administrativos que afecten a derechos o intereses de los particulares podrán conservarse en soportes de esta naturaleza, ya sea en el mismo formato a partir del que se originó el documento o en otro cualquiera que asegure la identidad e integridad de la información necesaria para reproducirlo. Se asegurará en todo caso la posibilidad de trasladar los datos a otros formatos y soportes que garanticen el acceso desde diferentes aplicaciones.

Los medios o soportes en que se almacenen documentos, deberán contar con medidas de seguridad que garanticen la integridad, autenticidad, confidencialidad, calidad, protección y conservación de los documentos almacenados. En particular, asegurarán la identificación de los usuarios y el control de accesos, así como el cumplimiento de las garantías previstas en la legislación de protección de datos".

\section{Obligación de las adminis-}

\section{traciones}

La Ley no determina cómo una organización debe o puede abordar la manera de hacer efectiva la relación con los ciudadanos por medios electrónicos; pero pone muchísimo énfasis en la utilización de las nuevas tecnologías en el funcionamiento interno, especialmente en la consecución de la tramitación electrónica: 
En cuanto al funcionamiento interno de la Administración, las nuevas tecnologías permiten oportunidades de mejora (eficiencia y reducción de costes) que hacen ineludible la consideración de las formas de tramitación electrónica, tanto para la tramitación electrónica de expedientes, como para cualquier otra actuación interna de la Administración, expandiéndolas gradualmente con el objetivo del año 2009. (Exposición de motivos III)

En definitiva, la estrategia de gestión de documentos electrónicos es un elemento esencial para garantizar el cumplimiento de la futura ley:

La tramitación electrónica conlleva la gestión de documentos electrónicos. La propia ley insiste en este aspecto:

"Las Administraciones Públicas utilizarán las tecnologías de la información de acuerdo con lo dispuesto en la presente Ley, asegurando la disponibilidad, el acceso, la integridad, la autenticidad, la confidencialidad y la conservación de los datos, informaciones y servicios que gestionen en el ejercicio de sus competencias. (Título preliminar, art. I.2)

¡Aunque puedan quedar dudas de que las administraciones públicas puedan conseguirlo íntegramente a corto o medio plazo, la aplicación de la ley nos empuja hacia el establecimiento del sistema de gestión de documentos electrónicos como soporte de las tramitaciones ya sean las comunicaciones con el ciudadano vía papel o por medios electrónicos. Esto equivale a establecer que los documentos "oficiales" de la Administración sean los electrónicos. Supone que los recibidos en papel deben digitalizarse adecuadamente para formar parte de los expedientes y que de la gestión y tramitación desaparecerían los documentos en papel.

Es posible que el sistema de gestión de documentos electrónicos requiera más adelante desarrollos reglamentarios. Mientras esto sucede las opciones tecnológicas existentes nos permiten que los documentos en los que se refleja la actividad de las administraciones públicas:

a) Se archiven (o guarden) dispersos en múltiples aplicaciones de gestión de datos o de expedientes.

b) Se archiven en un repositorio único y organizado.

c) Desaparezcan como "objetos documento" y se conviertan en datos de las aplicaciones de gestión.

En cada Administración estas decisiones estratégicas deben tomarse de acuerdo con las capacidades y medios de cada una de ellas, de forma que se puedan obtener los máximos beneficios, evitando los riesgos.

4. Por qué se hace más necesaria una estrategia de gestión de documentos

La gestión de documentos siempre ha sido necesaria, tanto en el mundo en papel como para los documentos electrónicos. Pero si bien en la gestión de los documentos en papel existe un conocimiento colectivo de buenas prácticas, que viene del uso del mismo desde hace algunos siglos, cuando hablamos de gestión de documentos electrónicos existen muchos procedimientos y prácticas menos conocidos y un montón de dudas. Es más necesario que nunca un modelo global que se aplique a toda la organización y que no puede ser una copia del modelo en papel. Cualquier modelo debe tener:

- un componente conceptual que implica la definición de cómo se quiere trabajar.
- un componente organizativo que establece las políticas, procedimientos y sistema de responsabilidades.

- un componente tecnológico que incluye las herramientas a utilizar, sus características, los nuevos procesos documentales (como la migración de soportes o la conversión de formatos), las interrelaciones con otras aplicaciones y la interoperabilidad.

Cualquier estrategia de gestión de documentos electrónicos debe basarse en los siguientes principios:

a) Conservar las evidencias y pruebas de las acciones de la organización en el corto, medio y largo plazo.

En casi todas las administraciones públicas existe una práctica administrativa con siglos de tradición respecto a la formación, organización y conservación de expedientes y documentos en papel. Aunque con bastantes dificultades, que en parte provienen de la propia aparición de las nuevas tecnologías en los hábitos de trabajo, de cierto deterioro de la importancia concedida a los archivos en las administraciones públicas y de la escasez de recursos, los sistemas de archivo tradicionales se han mantenido a lo largo del tiempo. En el entorno electrónico, las instituciones deberán poder conservar sus documentos a largo plazo con las mismas garantías de autenticidad, integridad y accesibilidad que se conservan en papel y esto supone una redefinición importante de funciones y competencias.

b) Facilitar el acceso y el entendimiento de los documentos tanto a los ciudadanos en los trámites que les afectan, como a los organismos o instituciones que los requieran como prueba de la acción administrativa e internamente dentro de las propias dependencias de las instancias gestoras. 
En los documentos en papel esta labor se ejerce mediante el establecimiento de procedimientos y políticas, y el cumplimiento de las mismas por el personal que custodia los documentos y expedientes. En los documentos electrónicos esto no es suficiente. Si los documentos y expedientes se encuentran en una tecnología a la que no se tiene acceso o que requiere de unas habilidades especiales para su comprensión se estarían limitando los derechos legítimos. Es necesario por lo tanto establecer los mecanismos por los que los documentos electrónicos se hacen accesibles y comprensibles por cualquier persona que tenga derecho a acceder a los mismos, sin necesidad de que dichas personas tengan que saber manejar o estar autorizadas a entrar en las aplicaciones en las que se gestionan los trámites administrativos.

c) Garantizar el cumplimiento de los requerimientos de autenticidad, integridad y confidencialidad de los documentos.

Estos requerimientos que en el mundo en papel son evidentes, son más complejos y novedosos cuando hablamos de documentos electrónicos. La estrategia de gestión documental debe establecer y velar por el cumplimiento de los requisitos de fiabilidad e integridad de los documentos. Tiene que incluir nuevos procedimientos y prácticas informáticas que los sistemas de validación y firma electrónica, la auditoría de los sistemas informáticos o la captura de metadatos asociados a los documentos. La complejidad tecnológica que pueden suponer no debe nunca comprometer los otros principios de la gestión de documentos, encontrando el equilibrio necesario entre todos ellos.

d) Garantizar la accesibilidad a medio y largo plazo de las evidencias electrónicas.

La necesidad de acceso a documentos y expedientes no se limita en el tiempo a la duración del trá- mite. Las administraciones deben conservar sus documentos durante el tiempo que de ellos se deriven derechos y obligaciones, para la consulta de antecedentes, para respaldar la responsabilidad durante un período determinado y para la investigación histórica.

\section{Qué hay que plantearse} ante la sustitución del papel por documentos electrónicos

Las administraciones públicas deben ser conscientes de la necesidad de plantearse una estrategia global de gestión de documentos electrónicos para no tener que dar soluciones individualizadas a cada situación particular.

Sin embargo lo habitual es que la implantación de la administración electrónica se haga por trámites o procesos concretos, empezando por los que son más demandados por la sociedad. En muchos casos esta automatización de procesos se hace sin reparar en la necesidad de abordarlo conjuntamente con la gestión de los documentos electrónicos que se van a producir.

\section{Problemática de la documentación}

Cuando el objetivo es que en esos procesos los documentos electrónicos sustituyan definitivamente al papel se deben plantear las siguientes cuestiones:

1. Qué documentos o información constituyen la evidencia del proceso

- ¿Qué documentos se producen en el proceso?

- ¿Cómo es el expediente en papel que se quiere sustituir?

- Una vez acabado el trámite ¿que información y documentos se necesitan para que constituya el expediente electrónico?

- ¿En que momento los documentos o la información se puede considerar definitiva y auténtica?
- ¿Qué documentos deberíamos convertir en papel (copia certificada) si el ciudadano exige el documento en papel?

- ¿A que datos y documentos debería acceder un ciudadano que tiene derecho a consultar el expediente electrónico?

La determinación de qué es documentación corporativa o evidencias es el paso previo imprescindible para delimitar el ámbito sobre el que actuar. Las evidencias pueden guardarse en forma de objetos-documentos (por ejemplo un pdf) o en forma de conjuntos de datos que representan la evidencia documental, siempre que se puedan cumplir las condiciones de disponibilidad, acceso, integridad, autenticidad, confidencialidad y conservación.

2. Cómo se puede asegurar la integridad y autenticidad de los documentos

- ¿La información o los documentos pueden eliminarse o modificarse?

- ¿Cuáles son los elementos de autentificación que se utilizan?

- ¿Cómo puede comprobar la autenticidad una persona con derechos que accede al expediente electrónico?

- ¿Pueden machacarse los datos de una aplicación una vez terminado el trámite?

- ¿Se guardan en formatos que impidan la escritura?

Asegurar estas características para la documentación de cada proceso que se haya determinado como evidencia es imprescindible para poder asegurar una buena gestión. Las formas para asegurarlas pueden ser diversas y pueden estar determinadas por los formatos, los lugares de almacenamiento, las firmas electrónicas o el blindaje de los datos.

3. Qué información asociada se guarda junto con los documen- 


\section{tos para hacerlos comprensibles y localizables}

- ¿Se guarda información con cada documento del proceso al que corresponde?

- ¿Se guarda información sobre el documento que nos permita identificar la fecha en que se reali$\mathrm{za}$, quien lo hace y las características básicas del mismo?

- ¿Se podrían identificar las personas que aprueban los documentos y los cargos que ejercen en ese momento?

- ¿Los tipos de documentos se han definido específicamente o se han alineado con otros sistemas?

- ¿Se han dado reglas para la cumplimentación de datos que responden a algún estándar (Nombres, DNI, Callejeros, Unidades administrativas, etc.)?

- ¿La información o datos que describen el documento son legibles por sí mismos sin necesidad de uso de la aplicación en la que se ha gestionado el documento o están generados a base de códigos?

- ¿Los documentos mantienen su vinculación, por ejemplo la pertenencia al mismo expediente? ¿Se guarda un índice del expediente?

- ¿Se podrá vincular automáticamente este documento con otros relacionados (por ejemplo dos expedientes de un mismo ciudadano, de la misma calle)?

Para manejar y gestionar un sistema documental es imprescindible contar con una estructura de clasificación, que es la base conceptual para tener la documentación identificada y organizada en categorías que representan las funciones y actividades de la organización a la que pertenecen.

Para hacer los documentos recuperables, legibles y fáciles de buscar -estén donde estén-, será necesario contar con elementos de descripción de los mismos (meta- datos) que sean lo más homogéneos posible. Cuando se analiza un proceso de forma aislada es difícil saber cuál es el camino a seguir, pero al menos se debería documentar cuáles decisiones se han tomado $\mathrm{y}$ por qué.

\section{Cómo se plantea el acceso a} los documentos en el presente y a lo largo de todo el ciclo de vida en que deban conservarse

- ¿Es posible que personas que tienen que tener acceso a los documentos -internas o ciudadanospuedan acceder a los mismos sin tener que usar las aplicaciones de tramitación?

- ¿Pueden las oficinas gestoras determinar a su criterio quién tiene que tener o no acceso a los documentos electrónicos?

- ¿los documentos pueden ser legibles, e inteligible su contexto fuera de la aplicación en la que se gestionan?

- ¿Cómo se define el nivel de seguridad que tienen que tener los documentos: cuáles están sujetos a Ley de Protección de Datos, cuáles son públicos, cuáles son confidenciales, a cuáles acceden los interesados?

- ¿Cómo se van a facilitar los accesos de los ciudadanos a los expedientes cuando lo requieran..., por internet, teniéndose que personar físicamente?

- ¿Quién y dónde puede emitir duplicados de los documentos digitales?

- ¿Quién y cómo se envían documentos electrónicos o copias de los mismos a instancias judiciales $\mathrm{u}$ organizaciones fiscalizadoras?

Un requerimiento esencial de la gestión documental es que los documentos sean accesibles cuando se requieran para distintos fines. Las decisiones sobre el nivel de acceso y seguridad que tienen que tener los documentos hay que establecerlas no sólo pensando en las necesidades del día a día de las oficinas, sino desde la necesidad de acceso para la organización, el ciudadano y la sociedad a medio y largo plazo. Hay que determinar cómo se va a garantizar que la documentación sea accesible (localizable, legible y disponible según los criterios de seguridad) a lo largo del tiempo. El acceso a los documentos tiene distintos componentes que se entremezclan: los formatos de conservación (los formatos abiertos como PDFa o OAIS parece que son ya un acuerdo internacional) y las estrategias de conservación (ver siguiente punto) por un lado; la capacidad para dar acceso electrónico a los que tienen derecho sin necesidad de conocimientos especiales; y la seguridad y protección de los datos confidenciales.

5. Cómo se plantea la conservación y almacenamiento de los documentos

- ¿Se ha planteado la eliminación de información cuando ya no se necesite?

- ¿Se plantea el crecimiento infinito de los datos?

- ¿Se plantean estrategias de migración de la información a otros soportes y/o sistemas cuando se necesite menos?

- ¿Se podrá acceder a los documentos y datos cuando ya no exista la aplicación?

- ¿Cómo se garantiza que los documentos a conservar no se pierden, o no han sido manipulados y son auténticos, íntegros y fiables?

- ¿Existen estrategias planificadas de conversión de datos a formatos de conservación?

Pensar en todos estos temas requiere un esfuerzo de proyección a futuro, aunque no sea muy lejano. Este es un punto muy relacionado con el anterior, y por lo tanto es difícil hacerlo de forma parcial. En este caso al menos la Admi- 
nistración se debería encaminar a minimizar los riesgos: los formatos abiertos, las tecnologías abiertas y los planteamientos de crecimiento no infinito de las aplicaciones son estrategias internacionalmente aceptadas. Es importante en este punto no confundir las copias de seguridad de las aplicaciones con la conservación de la información y los documentos.

6. Qué rastro queda de las operaciones realizadas sobre los documentos
- ¿Queda constancia del uso de la información o los documentos?

- ¿Queda constancia de los procesos de importación, exportación o traspaso a otro almacenamiento?

Con los documentos electrónicos la trazabilidad de las acciones realizadas sobre los documentos es posible técnicamente. Estos datos permiten hacer frente a posibles auditorías que demuestren las características que pedimos a los documentos.

\section{Nota:}

1. El proyecto en un principio se llamó Ley para el acceso electrónico de los ciudadanos a las administraciones públicas (Laecap)

Carlota Bustelo; Elisa García-Morales, Inforárea SL, Núñez de Balboa, 118, 1-D. 28006 Madrid.

Tel.: +34-915 971 383; fax: +34915970582

cbustelo@inforarea.es

correo@inforarea.es

\section{E-DOCPA}

\section{edocpa@congresosasturias.com http://edocpa.com/}

\section{El Parlamento Europeo considera que a partir de las bibliotecas digitales y los repositorios institucionales se puede construir una red para el intercambio de conocimientos y de cultura, próxima a todos los ciudadanos, y una de cuyas tareas básicas es poner al alcance y servicio de la ciudadanía la información necesaria para el desenvolvimiento

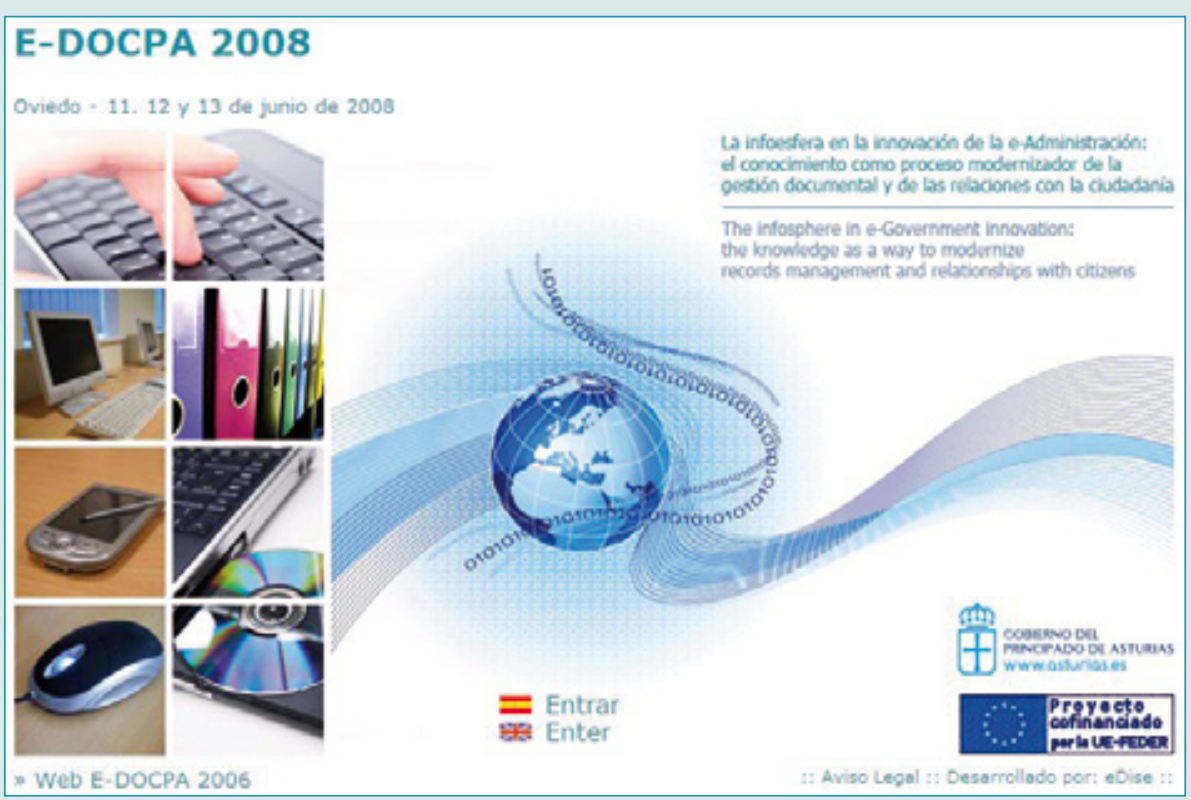 social, con independencia del lugar de residencia, el nivel de formación o la situación económica.}

